Check for updates

Cite this: RSC Adv., 2019, 9, 16406

\title{
Hyper-branched structure-an active carrier for copolymer with surface activity, anti- polyelectrolyte effect and hydrophobic association in enhanced oil recovery $\dagger$
}

\author{
Qi Wu, ${ }^{\text {ac }}$ Shaohua Gou, (DD *ab Jinglun Huang, ${ }^{\star c}$ Guijuan Fan, ${ }^{c}$ Shiwei Li ${ }^{\mathrm{a}}$ \\ and Mengyu Liu $^{\mathrm{a}}$
}

Herein, a hyper-branched polymer h-PMAD with, simultaneously, surface activity, an anti-polyelectrolyte effect and a hydrophobic association was prepared via aqueous solution free radical polymerization, and characterized by IR, NMR, TG-DTG and SEM. The polymer h-PMAD provided excellent comprehensive properties in terms of surface activity, thickening, water solubility, rheology and aging, which were compared with studies of HPAM and the homologous linear polymer PMAD. Specifically, the IFT value was $55.40 \mathrm{mN} \mathrm{m}^{-1}, 789.24 \mathrm{mPa}$ s apparent viscosity with a dissolution time of $72 \mathrm{~min}, 97.72,90.77$ and $105.81 \mathrm{mPa}$ s with $\mathrm{Na}^{+}, \mathrm{Ca}^{2+}$ and $\mathrm{Mg}^{2+}$ of 20000 , 2000 and $2000 \mathrm{mg} \mathrm{L}^{-1}$, respectively. Meanwhile, the non-Newtonian shear thinning behavior had a $96.33 \%$ viscosity retention while the shear rate went from $170 \mathrm{~s}^{-1}$ to $510 \mathrm{~s}^{-1}$ and then returned to $170 \mathrm{~s}^{-1}$ again and $0.12 \mathrm{~Hz}$ curve, with an intersection frequency of $G^{\prime}$ and $G^{\prime \prime}$. Also, it had $33.51 \%$ and $50.96 \%$ viscosity retention in formation and deionized water at $100{ }^{\circ} \mathrm{C}$ and a low viscosity loss in formation water at $80{ }^{\circ} \mathrm{C}$ over 4 weeks. Moreover, the h-PMAD had an EOR of $11.61 \%$, was obviously higher than PMAD with $8.19 \%$ and HPAM with 5.88\%. Most importantly, the better EOR of h-PMAD over that of PMAD testified that the hyper-branched structure provided an active carrier for copolymers with functionalized monomers to exert greater effects in displacement systems, which is of an extraordinary meaning.

Received 1st March 2019
Accepted 10th May 2019
DOI: 10.1039/c9ra01554j
rsc.li/rsc-advances phase separation and precipitation by flocculation destroyed the displacement and profile control performance; ${ }^{7-9}$ (c) a high temperature resulted in the conversion of amide into carboxylic acid groups, hydrolysis and thermal oxidative degradation, which caused the disruption of polymer backbones, and, moreover, shearing weakened the hydrogen bond and so increased the sensitivity to formation water, resulting in a significant drop in viscosity, which greatly affected the stability of its fluidity. ${ }^{10-12}$

In response to above shortcomings in HPAM, many oilfield researchers carried out many works to find excellent performance alternatives for the new reservoir environment, including the introduction of functional groups and new molecular structures. On the one hand, hydrophobically associated acrylamide copolymers had gained extensive focus due to their unique rheological properties. ${ }^{13-15}$ Liu et al. ${ }^{16}$ reported a hydrophobically-associated poly(acrylic acid-co-acrylamide) gel prepared via micellar polymerization, which showed a superior mechanical stability and convincing phase transition behavior. Also, a star-shaped nanosilica core-shell twin-tailed hydrophobic association copolymer, SHPAM, with a strong core compatibility and fluidity control ability for polymer flooding in harsh environments, was synthesized by Pu et al. ${ }^{17}$ enhancing the oil recovery to reach $20 \%$. On the other hand, the zwitterionic polymers are also widely used in oilfield development due to their remarkable hydration ability, salt
Chengdu 610500, P. R. China.E-mail: shaohuagou@swpu.edu.cn

${ }^{b}$ State Key Laboratory of Oil and Gas Reservoir Geology and Exploitation, Southwest Petroleum University, Chengdu 610500, P. R. China

'Institute of Chemical Materials, China Academy of Engineering Physics, Mianyang 621900, China. E-mail:jinglunhuang@163.com

$\dagger$ Electronic supplementary information (ESI) available. See DOI: 10.1039/c9ra01554j 
resistance, high mechanical stability and temperature responsiveness, thanks to their anti-polyelectrolyte effect. ${ }^{18-20}$ The presence of free ions shielded the mutual attraction between inner chains in molecules and enhanced the bonding of molecules to each other, so that with an increasing hydrodynamic volume, the molecular conformation became more stretched, resulting in an increase in viscosity instead of falling. ${ }^{21,22}$ Zuniga C. A. et al. ${ }^{23}$ obtained a novel system, I-prGO, on the partially reduced graphene oxide functionalized sulfonate-based zwitterionic polymer poly(vinylimidazole)-co-poly(aminostyrene), which could be used as a carrier for molecular delivery and as a protective inclusion of nanomaterials, and also for reservoir mapping and enhanced oil recovery. For example, the copolymer exhibited a highly stable dispersion after 140 days at $90^{\circ} \mathrm{C}$ in high concentrations of Arab-D and API from deep reservoirs. Holtmyer M. D. et al. ${ }^{24}$ synthesized a ternary zwitterionic polymer AM-AMPS-MAPDMDHPAS as a fracturing fluid thickener by inverse emulsion polymerization with a better temperature resistance $\left(>176.7^{\circ} \mathrm{C}\right)$ and salt resistance (3000 $\mathrm{mg} \mathrm{L}^{-1}$ ) than HPAM, suitable for operation in high temperature and high salinity acidified fracturing formations. In addition, the zwitterionic polymers also acted as a surfactant, which could reduce the interfacial tension and increase the sweep coefficient to improve the oil displacement efficiency.

For several years, dendritic highly branched macromolecules had shown a series of unique physical and chemical properties, such as excellent solubility, high rheology, low viscosity, and a large number of modifiable terminal functional groups that are not possessed by linear polymers due to their three-dimensional spherical and highly branched structures. ${ }^{25}$ Among these are the hyper-branched polymers that performed several unique application prospects in the fields of controllable macroscopic materials, sustained release drug carriers, information storage materials, high-efficiency catalysts, nonlinear optical materials, nanomaterials, water treatment, separation membranes, rheology modifiers, etc. ${ }^{26-29}$ Meanwhile, the application to research in oil fields has also gradually become active. Wever D. A. Z. et al. ${ }^{30}$ reported that the linear, star and comb polyacrylamides (PAMs) prepared via atom transfer radical polymerization (ATRP) in an aqueous medium at room temperature, investigated the effects of molecular structure on aqueous rheology, including viscosity and elasticity, paving the way for the application of these polymeric materials in enhanced oil recovery. $\mathrm{Pu}$ et $a .^{31}$ synthesized amphiphilic hyper-branched polymers AMHPM that respond to shear, temperature, salt and $\mathrm{pH}$ by effectively controlling the hydrodynamic size of the terminal functional groups of monomers. More, novel star-like polymers (SLPs) with a four-gradient molecular weight, consisting of nano-silica as the core and a layer of hydrophilic chains as the shell, were made via mild water free-radical polymerization. The study showed that rigid nuclei with the three-dimensional microstructures of SLPs had ideal performances in thickening and long-term stability together, which were instructive for the use of hyper-branched polymers for oil recovery. ${ }^{32}$ Klemm B. J. et al..$^{33}$ probed the suitability of a series of star-branched polyacrylamides (SB-PAMs) in EOR. The comparison demonstrated that the SB-PAMs presented a higher robustness under shear and a higher salt tolerance; contrary to HPAM, the salinity did not influence the solution viscosity of the SB-PAMs.
Moreover, they were approximately 3-5 times better than their linear analogues with similar molecular weights at EOR tests and showed more resilience under harsh reservoir conditions, which made them attractive for EOR applications.

Based on the above research, it can be concluded that the introduction of special groups, such as hydrophobic groups and ionic groups, and the construction of special molecular structures, such as core shells and branch in polymer molecules, are both effective ways to improve the performance of HPAM, which achieved significant results. However, the problem was generally that the molecules which introduced excellent groups had normal structures, and the intra-molecular functional groups of the good structures were monotonous. Obviously, it is undoubtedly a good choice to combine the advantages of the two parts with the same polymer molecule so as to further improve the overall performance, but the related research was relatively rare.

In our previous works, we prepared a hydrophobically associating polyacrylamide based on oleic imidazoline and sulfonate, ${ }^{34}$ an anti-biodegradable hydrophobic sulfonate-based acrylamide copolymer containing 2,4-dichlorophenoxy, ${ }^{35}$ an aaminophosphonic acid-modified acrylamide-based hydrophobic-associating copolymer ${ }^{36}$ and complexes of hydrophobically modified polymer and surface active imidazoliumbased ionic liquids, ${ }^{37}$ which all showed gratifying results in enhanced oil recovery, providing strong evidence for the excellent properties of hydrophobic associations and ionic polymers. Based on these results, we made a new attempt to prepare a comb-shaped polyzwitterion with surface-activity via a $\mathrm{N}$ maleoyl chitosan modified HPAM, the surface tension decreased with the increase of polymer solution concentration within a certain range, this discovery had a great positive meaning on overcoming the chromatographic separation effect and reducing the oil-water interfacial tension to improve oil recovery, also introduced comb-shaped model in copolymer structure. ${ }^{38}$ Combining with these works, in this paper we selected a special structure of maleated triethylenetetramine $(\mathrm{N}$ TETA) with a hydrophobic monomer (DMBSA), zwitterionic monomer (MMPES), AM and AA, prepared a hyper-branched structural copolymer that exhibited surface activity, an antipolyelectrolyte effect and hydrophobic association simultaneously via aqueous solution free radical polymerization. The polymer structure was characterized by IR and NMR, thermal analysis by TG-DTG, and microscopic appearance by SEM. More, surface activity, thickening ability, water solubility, aging and rheological properties, including shear, salt and temperature resistance and viscoelasticity, were compared with HPAM and corresponding linear copolymers. Finally, the mobility control ability and EOR were studied via a core flooding experiment to discuss whether it had potential application in displacement systems.

\section{Experimental}

\subsection{Material}

Acrylamide (AM), acrylic acid (AA), p-toluenesulfonyl chloride (PTSC), polyoxyethylene octylphenol ether (OP-10), $N, N$ - 
diallylamine (DAP), triethylenetetramine, hydroquinone, triethylamine $\left(\mathrm{Et}_{3} \mathrm{~N}\right)$, ammonium persulfate $\left(\left(\mathrm{NH}_{4}\right)_{2} \mathrm{~S}_{2} \mathrm{O}_{8}\right)$, sodium bisulfite $\left(\mathrm{NaHSO}_{3}\right)$, dimethyl sulfoxide, dichloromethane, 1,4dioxane, acetone, hydrochloric acid, anhydrous sodium sulfate, sodium chloride, sodium hydroxide, absolute ethanol and acetonitrile (AR for all the above chemical reagents, from Chengdu Kelong Chemical Reagent Factory, Sichuan, China). $\operatorname{HPAM}\left(M_{\mathrm{w}}=1.5 \times 10^{7}\right.$, Daqing Refining and Chemical Company). $N$-(3-Dimethylaminopropyl) methacrylamide (DAPMAA), and 3-(methacryloyloxy) propyltrimethoxysilane (MPS) (CR for these chemical reagents, from Chengdu Huaxia Chemical Reagent Factory, Sichuan, China). Methylethylene sulfite (MES), monomers MMPES and DMBSA were prepared according to the literature $^{\mathbf{3 8 - 4 0}}$ (for details for MMPES and DMBSA see ESI $\dagger$ ).

\subsection{Synthesis of monomer $N$-maleoyl triethylenetetramine (N-TETA)}

Into a $100 \mathrm{~mL}$ flask, triethylenetetramine $(50 \mathrm{mmol}, 7.5 \mathrm{~mL})$ was dissolved in DMSO $(30 \mathrm{~mL})$ at room temperature with stirring until it became homogeneous. Then, a mixture of maleic anhydride (220 mmol, $21.57 \mathrm{~g}$ ) and DMSO was dribbled into the flask, and upon addition the temperature of the system was up to $60^{\circ} \mathrm{C}$. After stirring for $5 \mathrm{~h}$, the precipitant was collected from a large amount of acetone and washed three times, dried under vacuum at $45{ }^{\circ} \mathrm{C}$, to give the final product, a light brown yellow fluffy solid $N$-TETA (90\% yield) (scheme in Fig. S3†). ${ }^{1} \mathrm{H}$ NMR $\left(400 \mathrm{MHz}, \mathrm{D}_{2} \mathrm{O}\right): \delta 7.25(\mathrm{~s}, 2 \mathrm{H},-\mathrm{C}(\mathrm{O})-\mathrm{NH}-)$, $6.81(\mathrm{~m}, 4 \mathrm{H},-\mathrm{CH}=$ $\mathrm{CH}-\mathrm{COO}^{-}$), $6.63\left(\mathrm{dd}, 4 \mathrm{H},-\mathrm{CH}=\mathrm{CH}_{-} \mathrm{COO}^{-}\right), 3.49(\mathrm{t}, 4 \mathrm{H},-\mathrm{NH}-$ $\left.\mathrm{CH}_{2}-\mathrm{CH}_{2}^{-}\right), 3.20\left(\mathrm{t}, 8 \mathrm{H},-\mathrm{C}(\mathrm{O})-\mathrm{N}\left(\mathrm{CH}_{2}\right)-\mathrm{CH}_{2}^{-}\right)$.

\subsection{Synthesis of copolymer N-TETA/MMPES/AM/AA/DMBSA (h-PMAD)}

Into a $100 \mathrm{~mL}$ three necked flask, the appropriate amount of $\mathrm{N}$ TETA, MMPES, DMBSA, OP-10 and MPS were well mixed with $20 \mathrm{~mL}$ of DI water, and then the $\mathrm{AM}$ and $\mathrm{AA}$ were added in sequence. After stirring for $20 \mathrm{~min}$, sodium hydroxide solution was used to adjust the $\mathrm{pH}$ of the system. Next, the reactor was kept in a water bath at a certain temperature under nitrogen atmosphere for $15 \mathrm{~min}$, then the $\mathrm{NaHSO}_{3}-\left(\mathrm{NH}_{4}\right)_{2} \mathrm{~S}_{2} \mathrm{O}_{8}(1 / 1 \mathrm{~mol}$ ratio) initiator was added and dispersed rapidly, and the reaction was kept at a certain condition for $10 \mathrm{~h}$. Finally, the copolymer gel was precipitated with ethanol and dried in a $45{ }^{\circ} \mathrm{C}$ vacuum, retrieving the powdered sample of h-PMAD (Scheme 1). The synthetic methods and optimum synthesis conditions of polymer MMPES/AM/AA/DMBSA (PMAD) are displayed in ESI. $\dagger$

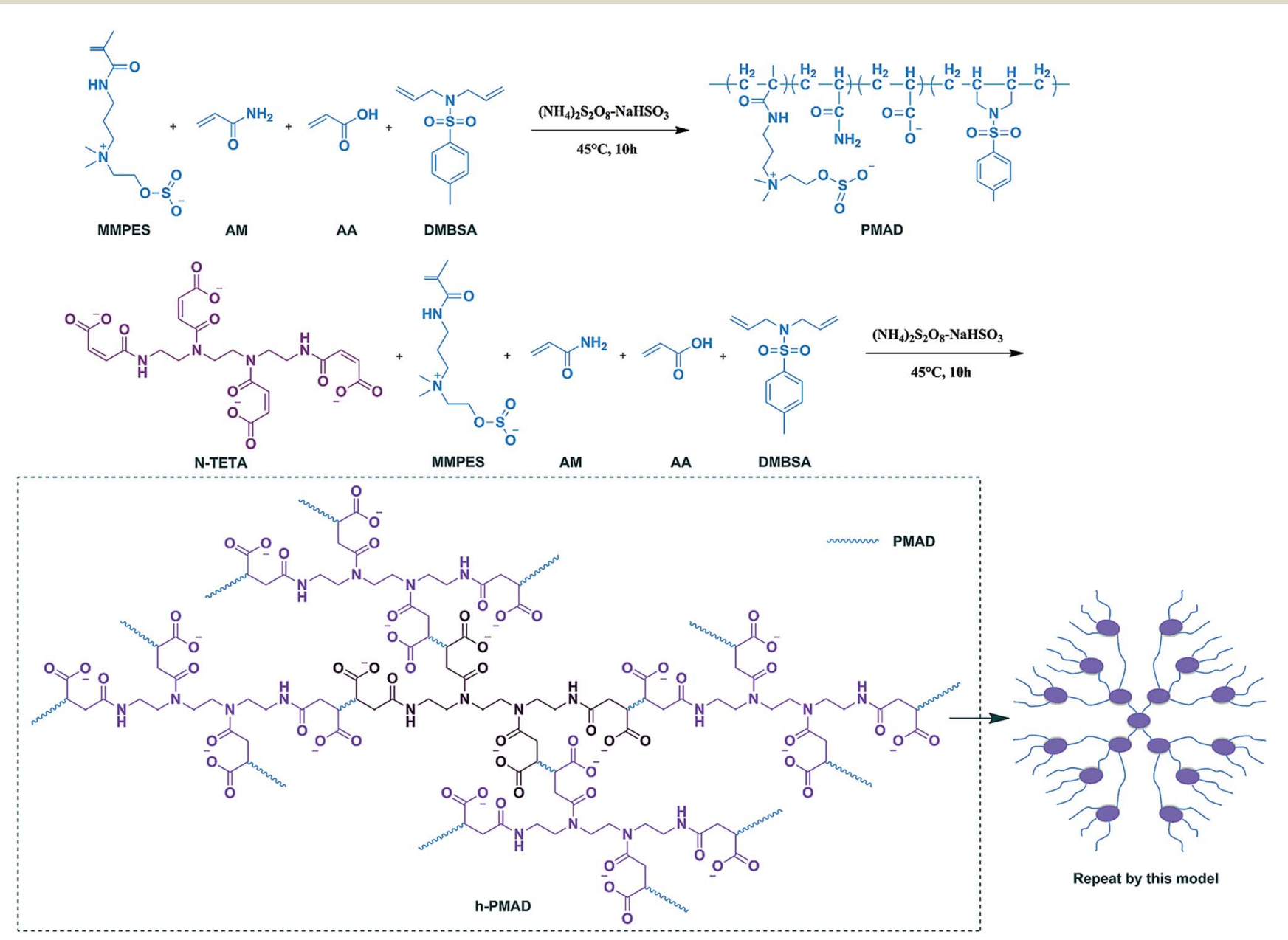

Scheme 1 The synthetic route of polymers. 


\subsection{Characterization}

2.4.1 IR. The Fourier-transform infrared (FT-IR) spectra of PMAD and h-PMAD with $\mathrm{KBr}$ pellets were scanned in the wavelength range of $4000-500 \mathrm{~cm}^{-1}$ by a WQF-520 Fouriertransform infrared spectrometer (Beijing Rayleigh Analytical Instrument Corporation, China).

2.4.2 ${ }^{1} \mathbf{H}$ NMR. The ${ }^{1} \mathrm{H}$ NMR spectra of the monomer MMPES, DMBSA, $N$-TETA, PMAD and h-PMAD were recorded on a BRUKER AM $400 \mathrm{MHz}$ Nuclear Magnetic Resonance Spectrometer (Bruker Co., Switzerland).

2.4.3 TG-DTG test. Thermal stability tests of the polymers were conducted and measured via a thermogravimetric analyzer (Switzerland, METTLER TOLEDD Co.) at a flow rate of 50 $\mathrm{mL} \min ^{-1}$ at $25-800{ }^{\circ} \mathrm{C}$ under a nitrogen atmosphere, with a heating rate for the TG experiments of $25{ }^{\circ} \mathrm{C}$ min.

2.4.4 SEM. The microscopic structures of the polymer solutions of HPAM, PMAD and h-PMAD were investigated via a scanning electron microscope (SEM). Samples were prepared via dropping a few drops of a $2000 \mathrm{mg} \mathrm{L}^{-1}$ polymer solution on a designated glass, which were frozen rapidly by liquid nitrogen. Last, the frozen samples were observed via a FEI Quanta 450 (FEI, USA).

\subsection{Thickening ability and water solubility}

The thickening ability was evaluated through the influence of polymer concentration on the apparent viscosity at $30{ }^{\circ} \mathrm{C}$. The water solubility was defined as the change in the conductivity of the polymer solution (2000 $\mathrm{mg} \mathrm{L}^{-1}$, white granular (20-35 mesh)/deionized water) obtained using a DDS-307 conductivity meter (Shanghai Rex Xinjiang Instrument Co., Ltd, China) at different dissolution times at $30 \pm 0.1^{\circ} \mathrm{C}$.

\subsection{Surface activity}

Surface activity was confirmed via a function of polymer concentration (in the simulated formation water) and interfacial tension (IFT) by a drop shape analyzer (DSA30, KRÜSS GmbH Co., Hamburg, Germany) at $30 \pm 0.1{ }^{\circ} \mathrm{C}$.

\subsection{Salt resistance}

Salt resistance was studied via adding several small molecule electrolytes (such as NaCl: $0-20000 \mathrm{mg} \mathrm{L}{ }^{-1}, \mathrm{MgCl}_{2} \cdot 6 \mathrm{H}_{2} \mathrm{O}$ and $\left.\mathrm{CaCl}_{2}: 0-2000 \mathrm{mg} \mathrm{L}^{-1}\right)$ to polymer solutions $\left(2000 \mathrm{mg} \mathrm{L}^{-1}\right)$ to characterize the apparent viscosities at $7.34 \mathrm{~s}^{-1}$ and $30{ }^{\circ} \mathrm{C}$.

\subsection{Rheological experiments}

The polymer $(0.2 \mathrm{wt} \%)$ rheological behavior was conducted by a HAAKE MARS III rheometer (HAAKE, Germany) at an appropriate temperature.

2.8.1 Shear thinning and shear recovery test. Shear thinning of $2000 \mathrm{mg} \mathrm{L}^{-1}$ polymer solutions was measured via a rotation rheometer (Haake RheoStress 6000, Germany) with a shear range of $0-200 \mathrm{~s}^{-1}$ at $25 \pm 0.1{ }^{\circ} \mathrm{C}$, and the shear recovery was performed with a stepped shear rate 170-510-170 $\mathrm{s}^{-1}$ under the same conditions as the others.
2.8.2 Viscoelasticity. Viscoelasticities of the polymer solutions (2000 $\mathrm{mg} \mathrm{L}^{-1}$ ) were obtained via measuring the elastic modulus $\left(G^{\prime}\right)$ and viscous modulus $\left(G^{\prime \prime}\right)$ with a particular stress $(1 \mathrm{~Pa})$ at $30{ }^{\circ} \mathrm{C}$ in the frequency scanning range $0.01-10 \mathrm{~Hz}$. The behavior of the viscoelasticity is described via eqn (1) and (2). ${ }^{41}$

$$
\begin{aligned}
& G^{\prime}(\omega)=G \frac{\omega^{2} \lambda^{2}}{1+\omega^{2} \lambda^{2}} \\
& G^{\prime \prime}(\omega)=G \frac{\omega \lambda}{1+\omega \lambda}
\end{aligned}
$$

Herein $G$ is the stress relaxation modulus, $\lambda$ is the relaxation time, and $\omega$ is the angular frequency.

2.8.3 Temperature resistance and long-term stability. The temperature resistance of polymers was measured by solution behavior at a $7.34 \mathrm{~s}^{-1}$ shear rate, with temperatures from $30{ }^{\circ} \mathrm{C}$ to $100{ }^{\circ} \mathrm{C}$ for $30 \mathrm{~min}$, and then keeping the $7.34 \mathrm{~s}^{-1}$ shear rate and $100{ }^{\circ} \mathrm{C}$ for another $30 \mathrm{~min}$. A long-term stability test was performed through measuring the apparent viscosity of $2000 \mathrm{mg} \mathrm{L}^{-1}$ polymer solutions (simulated formation water, details in Table $\mathrm{S} 5 \dagger$ ) at different times at $80^{\circ} \mathrm{C}$ for 4 weeks.

\subsection{Core flooding experiment}

The core flooding experiment aims to study the fluidity control ability of the prepared polymer and its improvement for oil recovery capacity. The core assembly was a stainless-steel cylinder packed with sand (80-100 mesh), the sand was washed with $30 \%$ hydrochloric acid to remove impurities and DI water was used to wash until the $\mathrm{pH}$ was 7 , and then dried under a vacuum environment. The simulation crude oil was collected from Shengli Oil Field (China) and the apparent viscosity was $61.5 \mathrm{mPa} \mathrm{s}$ at $70{ }^{\circ} \mathrm{C}$. The simulated formation water was injected at $2.5 \mathrm{~mL} \mathrm{~min}{ }^{-1}$ into the sand filling model until the pressure was stable to determine its porosity with Darcy's law, and then the crude oil was injected into the core assembly and aged for $36 \mathrm{~h}$ at $70{ }^{\circ} \mathrm{C}$. Core flooding experiments were performed with the following steps.

First, the simulated formation water was injected at 0.4 $\mathrm{mL} \min ^{-1}$ until the out liquid water ratio exceeded $98 \%$, and then changed to polymer solutions with the same velocity $(0.4$ $\left.\mathrm{mL} \min ^{-1}\right)$. Finally, the water ratio ( $\left.>98 \%\right)$ from the second flooding was driven by subsequent water flooding at 0.4 $\mathrm{mL} \mathrm{min}^{-1}$. EOR was calculated with eqn (3).

$$
\mathrm{EOR}=E_{1}-E_{2}
$$

where EOR is the enhanced oil recovery, $\% ; E_{1}$ is the polymer flooding recovery, $\% ; E_{2}$ is the water flooding recovery, $\%$.

The fluidity control ability was formulated by the resistance factor (RF) and residual resistance factor (RRF) through eqn (4) and (5), respectively. ${ }^{42}$

$$
\begin{gathered}
\mathrm{RF}=\frac{P_{1} / Q_{1}}{P_{0} / Q_{0}} \\
\mathrm{RRF}=\frac{P_{2} / Q_{2}}{P_{0} / Q_{0}}
\end{gathered}
$$


Herein $P_{0}, P_{1}$ and $P_{2}$ are the constant pressure of the first water flooding, polymer flooding and subsequent water flooding, MPa; $Q_{0}, Q_{1}$ and $Q_{2}$ are the constant injection rates of first water flooding, polymer flooding and subsequent water flooding, $\mathrm{mL} \mathrm{min}^{-1}$. All the polymer solutions were prepared with the simulated formation water.

\section{Results and discussion}

\subsection{Optimum conditions}

The polymer h-PMAD were prepared via a redox free radical polymerization initiated by $\left(\mathrm{NH}_{4}\right)_{2} \mathrm{~S}_{2} \mathrm{O}_{8}-\mathrm{NaHSO}_{3}$; the optimum synthesis conditions were a single factor control variable method and the results are displayed in Fig. 1 and Table 1 (for details see Tables S2 and S3†). It can be seen from Fig. 1 that changes in different factors can cause obvious changes in viscosity, the combination of the horizontal factor values corresponding to each peak was the optimal synthesis condition.

For monomers, it was generally believed that a smaller monomer concentration resulted in a lower molecular weight of the polymer, and an increase in concentration led to an increase in the probability of contact and collision between the monomers, which contributed to the growth of the molecular chain and an increase in the molecular weight. However, when the monomer concentration was increased to a certain extent, the heat released by the polymerization could not be easily removed, resulting in an excessive temperature rise and an accelerated decomposition rate of the initiator; the increase in
Table 1 The optimal synthesis conditions for polymer h-PMAD

Feed ratio $(\mathrm{g})$

\begin{tabular}{llllllll} 
AM & AA & $N$-TETA & MMPES & DMBSA & pH & Initiator $(\mathrm{g})$ & Tem. $\left({ }^{\circ} \mathrm{C}\right)$ \\
\hline 7 & 3 & 0.4 & 0.2 & 0.03 & 7 & 0.03 & 45
\end{tabular}

the number of active sites in the system reduced the molecular weight of the synthetic polymer. ${ }^{\mathbf{4 3 , 4 4}}$ In addition, the $\mathrm{pH}$ of the medium not only affected the reaction kinetics but the structure and properties of the polymer, such as the polymerization rate, solubility, molecular weight and sequence structure of the monomer, resulting in different apparent viscosities. For example, when the system was strongly alkaline, the polymer began to hydrolyze, which accelerated as the $\mathrm{pH}$ increased. ${ }^{45}$ Also, the amount of initiator determined the amount of free radicals in the polymerization system; low concentrations corresponded to a low initiation efficiency, and the monomer conversion rate improved with an increase in the amount added. However, when the concentration was overly high, too many radicals appeared, and the excessively rapid reaction caused a rapid increase in the viscosity, resulting in a gel effect, which forced a widening of the molecular weight distribution and the reduction of apparent viscosity. ${ }^{\mathbf{4 6 , 4 7}}$ Finally, according to the reaction kinetics, the relationship between the decomposition rate constant of the initiator and the temperature can be expressed by the Arrhenius formula, ${ }^{\mathbf{4 8}}$ and the decomposition
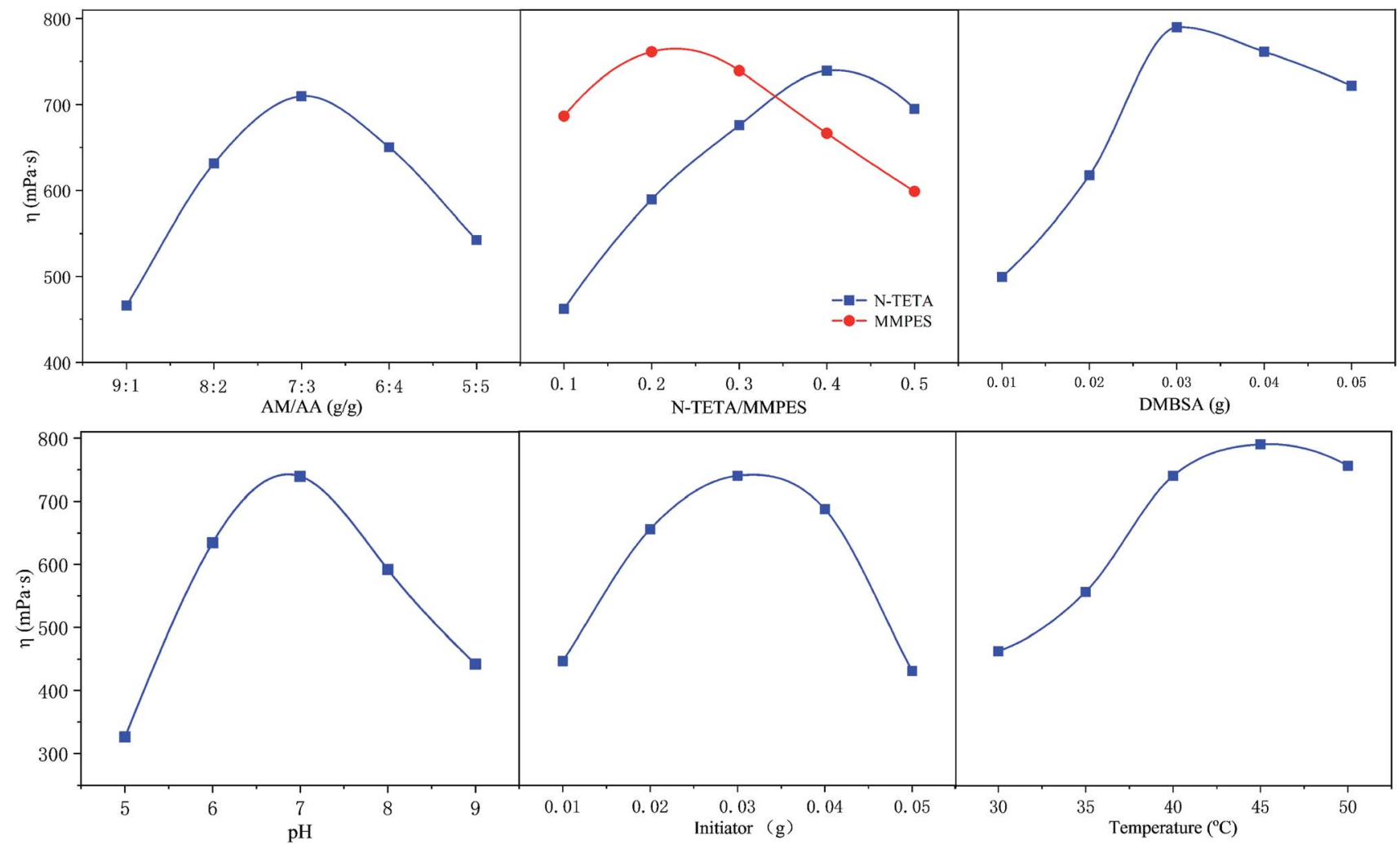

Fig. 1 The optimization of the synthesis conditions for polymer h-PMAD. 
rate increased with a rise in temperature, so the effect of temperature on the molecular weight and viscosity of the copolymer was similar to that of the initiator.

\subsection{IR and ${ }^{1} \mathrm{H}$ NMR}

The IR spectra of polymer PMAD and h-PMAD are shown in Fig. 2 and offer significant evidence on the chemical functional groups in materials. For h-PMAD, the characteristic peak at $3431 \mathrm{~cm}^{-1}$ was due to $-\mathrm{OH}$ in $\mathrm{R}-\mathrm{COOH}$, the peaks at 3261,1559 , and $1655 \mathrm{~cm}^{-1}$ were attributed to the $\mathrm{N}-\mathrm{H}$ and $\mathrm{C}=\mathrm{O}$ bonds, respectively, in the $-\mathrm{CONH}-$ group. The $\mathrm{C}-\mathrm{H}$ bonds of $\mathrm{R}-\mathrm{CH}_{3}$ and $-\mathrm{CH}_{2}-$ can be observed at $2923,2849,1456$ and $1390 \mathrm{~cm}^{-1}$. The peaks at $1317 \mathrm{~cm}^{-1}$ correspond to $\mathrm{C}-\mathrm{O}$ of the $-\mathrm{COO}-$ group. Moreover, the characteristic peaks at 1095 and $1044 \mathrm{~cm}^{-1}$ were confirmed as the stretching vibration of $\mathrm{S}=\mathrm{O}$ in the $\mathrm{R}^{-} \mathrm{OSO}_{2}-$ group, the peak at $712 \mathrm{~cm}^{-1}$ was due to $\mathrm{C}-\mathrm{S}$ in the $\mathrm{Ar}_{-} \mathrm{SO}_{2}$ - bond, and the absorption peak at $882 \mathrm{~cm}^{-1}$ was attributed to bending vibration of $\mathrm{C}-\mathrm{H}$ in the benzene ring structure. For PMAD, all the characteristic absorption peaks were similar to those of h-PMAD.

Fig. 3 illustrates the ${ }^{1} \mathrm{H}$ NMR spectra of polymers PMAD and $\mathrm{h}$ PMAD. For PMAD, the characteristic peaks at 1.11 and $3.59 \mathrm{ppm}$ correspond to absolute ethanol from the polymer purification, the chemical shift values at 8.39 and $7.13 \mathrm{ppm}$ were assigned to the proton peaks of the $-\mathrm{C}(\mathrm{O})-\mathrm{NH}-$ and $-\mathrm{C}(\mathrm{O})-\mathrm{NH}_{2}$ groups, respectively. The proton peaks of aromatic $\mathrm{Ar}-\boldsymbol{H}$ appeared at 7.71 and $7.41 \mathrm{ppm}$, and the proton peak of $-\mathrm{CH}_{2}-\mathrm{OSO}_{2}$ - bond was observed at $3.79 \mathrm{ppm}$. Peaks at 2.48, 2.14 and $1.58 \mathrm{ppm}$ were due to the $-\mathrm{CH}_{3},-\mathrm{CH}_{2}-$ and $-\mathrm{CH}$ - groups, respectively. For h-PMAD, all the proton peaks were similar to those of PMAD except the chemical shift value at $3.26 \mathrm{ppm}$, which was attributed to the -NH- group from $N$-TETA. According to the IR and ${ }^{1} \mathrm{H}$ NMR spectra, it can be judged that the structure of polymers PMAD and h-PMAD were in line with expectations.

\subsection{TG-DTG analysis}

The TG-DTG analysis of the two kinds of polymers is displayed in Fig. 4(a) and (b). There were four stages for the weight losses of polymers PMAD and h-PMAD with similar processes, attributed to the dehydration between intra-molecular \& intermolecular and the decomposition of the amide groups, the fracture of carbon-nitrogen bonds, the decomposition of the carbon-oxygen \& carbon-hydrogen bonds and the carbonization of the copolymers, respectively. Also, polymers PMAD and h-PMAD kept a good thermal stability until around $400{ }^{\circ} \mathrm{C}$ and had a higher mass retention rate of more than $40 \%$ at $800{ }^{\circ} \mathrm{C}$ compared with HPAM. ${ }^{49}$ Interestingly, though the final mass retention rate was similar, the hyper-branched polymer h-PMAD behaved with a much slower dehydration rate between intramolecular and inter-molecular than that of the linear polymer PMAD, which confirmed that the hyper-branched structure had a greater contribution to the thermal stability of the polymer molecules at a lower temperature, providing more possibilities for practical applications.

\subsection{Microcosmic configuration analysis by SEM}

The microcosmic appearances of HPAM (a), PMAD (b) and hPMAD (c) were studied via SEM, shown in Fig. 5. From Fig. 5(a), the micrograph of HPAM exhibited a loose network structure with larger pores. In contrast, the molecular chains of linear polymer PMAD (Fig. 5(b)) were more compact and showed ordered connections, mainly due to the hydrophobic monomer DMBSA in the polymer providing the association of molecular chains, forming more hydrophobic domains. Meanwhile, the zwitterions MMPES increased the electrostatic interaction between the molecular chains, the hydrophobic association and electrostatic interaction were much stronger than hydrogen bonding, leading to the existence of obvious advantages.$^{38}$ More importantly, from Fig. 5(c), the polymer hPMAD had a dense and hierarchical three-dimensional network structure while retaining the advantages of PMAD, the hyper-branched structure optimized the spatial arrangement of the linear molecular chains and had a better effect in enhancing the repulsion between carboxylate ions. ${ }^{50}$ This comparison of the microcosmic appearances demonstrates that a

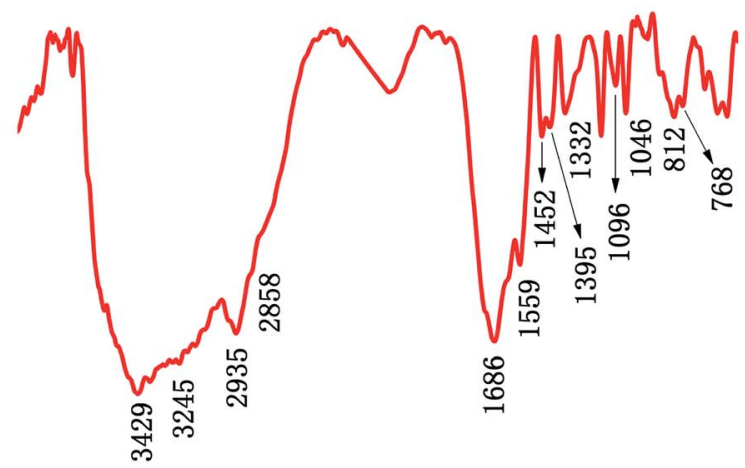

b

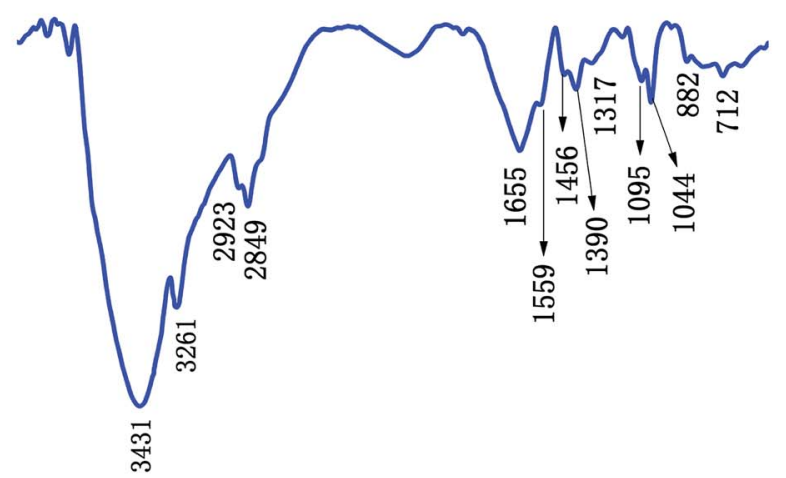

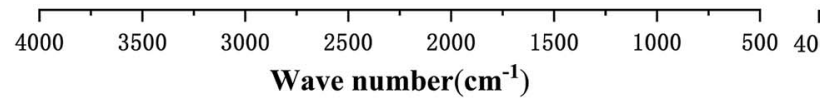

Wave number $\left(\mathrm{cm}^{-1}\right)$

Fig. 2 IR spectra of polymers: (a) PMAD, (b) h-PMAD. 


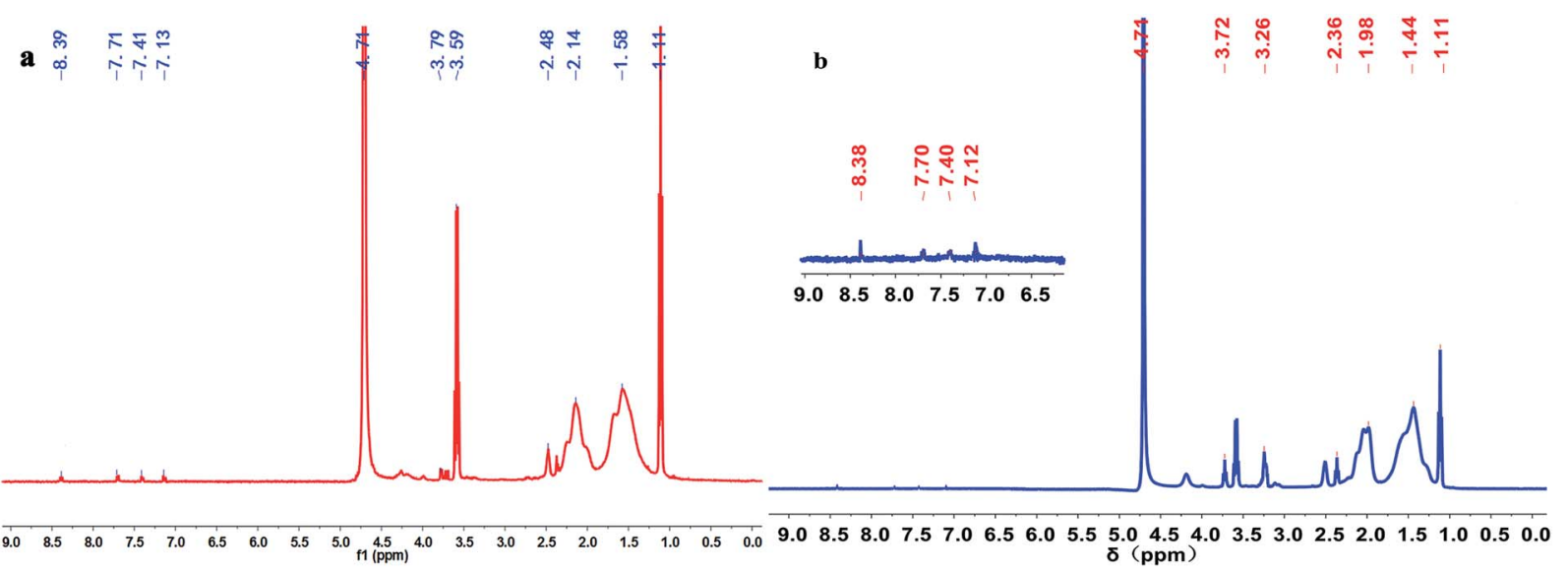

Fig. $3{ }^{1} \mathrm{H}$ NMR spectra of polymers: (a) PMAD, (b) h-PMAD.
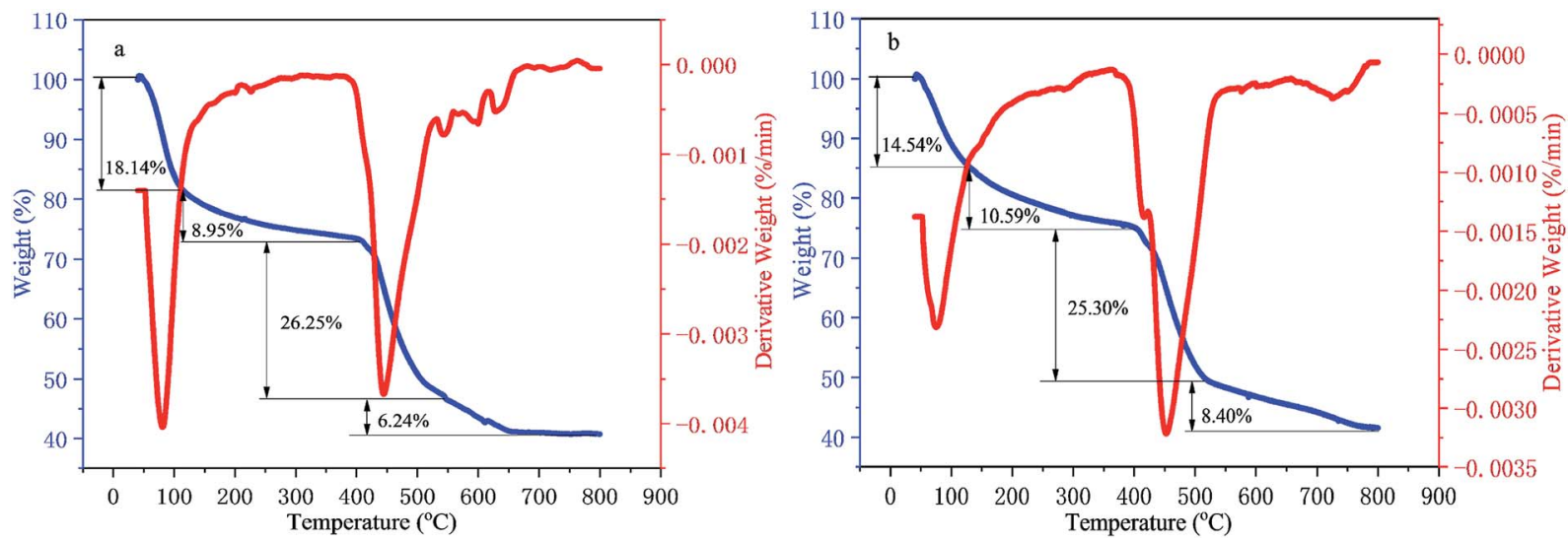

Fig. 4 TG-DTG curves of polymers: (a) PMAD, (b) h-PMAD.

polymer h-PMAD plays a potential role in rheological properties and displacement characteristics.

\subsection{Thickening ability and water solubility}

To investigate the relationship between the apparent viscosity and concentration, the apparent viscosity as a function of concentration in deionized water was determined for the polymers HPAM, PMAD and h-PMAD as displayed in Fig. 6(a) and (b). In general, the viscosity of these polymers increased with increasing concentrations but with significant differences; polymers PMAD and h-PMAD demonstrated a much superior thickening efficiency. As is shown in Fig. 6(b), when the concentration of polymer solution is lower than the critical association concentration (CAC), the growth rate of viscosity in HPAM was slightly higher than that in PMAD and h-PMAD, with a linear viscosity-concentration relationship. According to the literature ${ }^{35}$ under CAC the association of hydrophobic groups mainly occurs intra-molecularly, and the contribution to viscosity is far less than the electrostatic repulsion of carboxylate ions from HPAM. However, when the concentrations exceeded CAC, the viscosities of the PMAD and h-PMAD were much higher than that of HPAM, exhibiting a power-law exponential growth. Intuitively, the better thickening ability of PMAD over that of HPAM is due to the stronger electrostatic interaction from MMPES and intermolecular hydrophobic

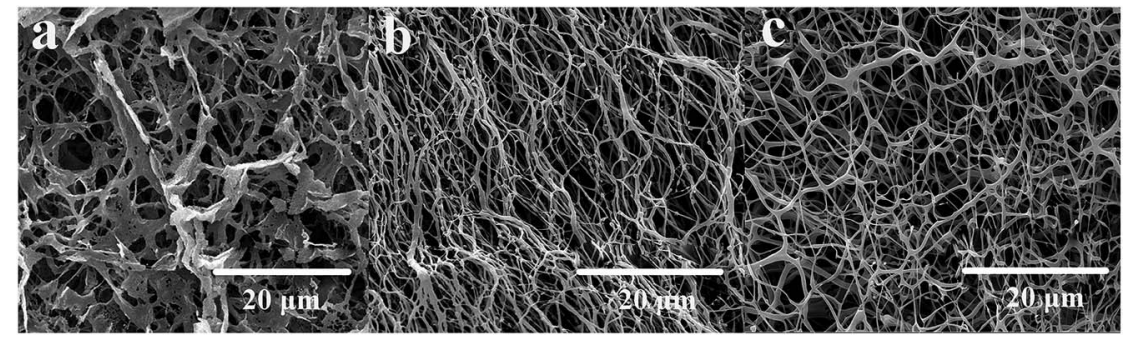

Fig. 5 SEM of polymers: (a) HPAM, (b) PMAD, (c) h-PMAD. 

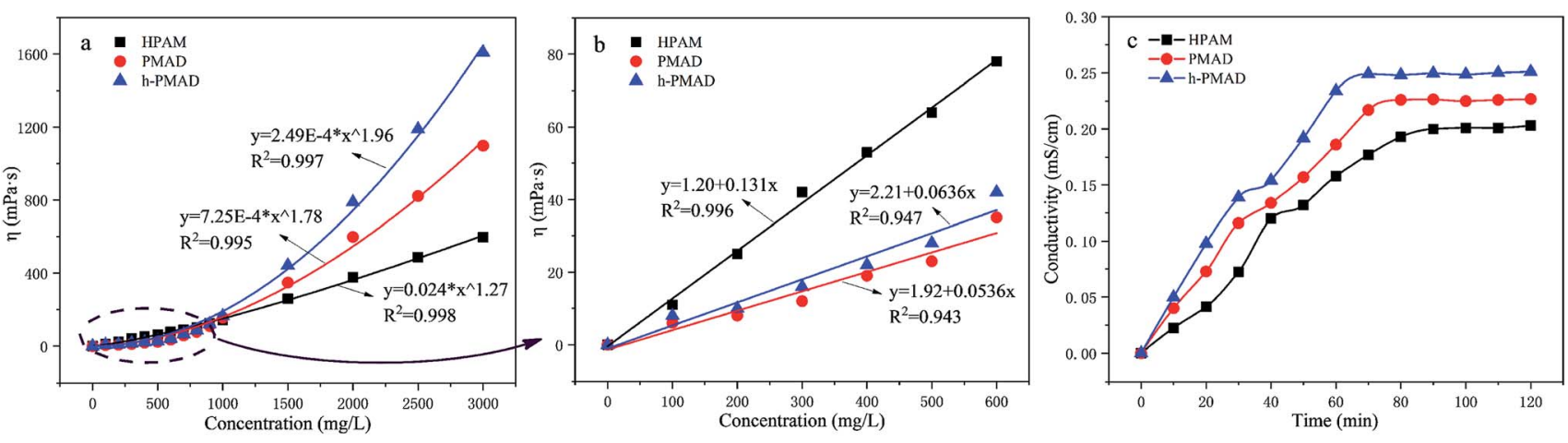

Fig. 6 ( $a$ and b) Tackifying of polymers. (c) The dissolution time curves of polymers.

association from DMBSA. More importantly, the hyperbranched structure enhanced the interaction between the polymer chains and exhibited a larger hydrodynamic volume, which led to an increase in viscosity ${ }^{32}$ so the polymer h-PMAD showed the highest thickening efficiency.

Objectively, a hyper-branched structure and the presence of hydrophobic groups could affect the solubility of the polymer, and poor solubility may lead to a bad performance in EOR. ${ }^{51}$ Fig. 6(c) shows the relationships between the conductivity and dissolution time; as we all know, higher conductivity values with the same dissolution time and a shorter steady time means a better solubility of the polymers. Obviously, polymers PMAD and h-PMAD exhibited more solubility at higher conductivity values all the time and a shorter steady time of $80 \mathrm{~min}$ and $72 \mathrm{~min}$, respectively, compared to HPAM (90 min), which is due to the presence of the carboxylate ion, and the quaternary ammonium and glycol sulfite from MMPES made the molecules more soluble. ${ }^{35}$ This theory only confirms why h-PMAD ha a shorter steady time than PMAD, originating from its large number of carboxylate ions.

\subsection{Surface activity}

The surface activity of the polymer HPAM, PMAD and h-PMAD solutions $\left(2000 \mathrm{mg} \mathrm{L}^{-1}\right)$ were investigated, as illustrated in Fig. 7. It was found that the interfacial tensions of PMAD and hPMAD were drastically reduced with an increase in concentration about $1000 \mathrm{mg} \mathrm{L}^{-1}$, and then tended to be steady at different steady states, and with different critical micelle concentrations (CMC) of $914 \mathrm{mg} \mathrm{L}^{-1}$ and $714 \mathrm{mg} \mathrm{L}^{-1}$, respectively. Obviously, the polymer h-PMAD solution showed the highest surface activity, the IFT reached a steady state at about $55.4 \mathrm{mN} \mathrm{m}^{-1}$, while HPAM remained around $71.8 \mathrm{mN} \mathrm{m}^{-1}$. It turned out that the polymers PMAD and h-PMAD all have the ability to reduce the interfacial tension, which provides a great positive effect on overcoming the effects of color-induced segregation, reducing the oil-water interfacial tension and thereby enhancing oil recovery. The good performance comes from the monomer MMPES, both anions and cations in the molecule all outside surface active, but there were marked differences in the polymers compared to the monomers; the polymer was slightly more surface active at low concentrations, and much higher than the monomer at the higher concentrations needed to obtain a substantial decrease of the surface tension. The surface tension decreased continuously in a nearly linear dependence on $\log$ [concentration], and thereby the higher molecular weight was beneficial for surface activity, consistent with the literature. ${ }^{52}$ Moreover, hydrophobic association in the polymer solutions formed molecular micelles that caused an increase in the interaction between the molecular chains and the reduction of the interfacial tension..$^{53}$

\subsection{Salt resistance}

The influence of salt on the three kinds of polymer solutions (2000 $\mathrm{m} \mathrm{L} \mathrm{L}^{-1}$ ) were measured at different salt concentrations, and the trend of the apparent viscosity to salt concentrations is shown in Fig. 8. In Fig. 8(a) and (b), the apparent viscosities of all the copolymer solutions decreased with an increase of salt concentrations. In the first stage, a sharp drop in apparent viscosity occurred; with an increasing salt concentration, slight decreases in the solutions' apparent viscosity were observed. Especially, the apparent viscosities of the PMAD and h-PMAD solutions were always significantly higher than that of HPAM, which was due to the effects of the anti-polyelectrolyte from the zwitterionic monomer MMPES. According to the literature, ${ }^{15}$ in a zwitterionic polymer aqueous solution, the molecular chain is curled because of the mutual attraction of anionic and cationic

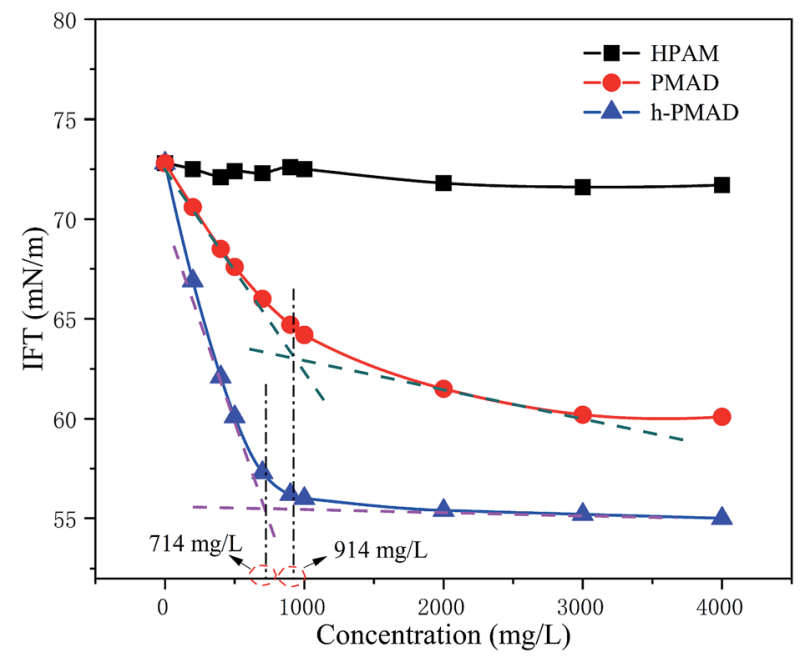

Fig. 7 Surface activity behavior of polymers. 

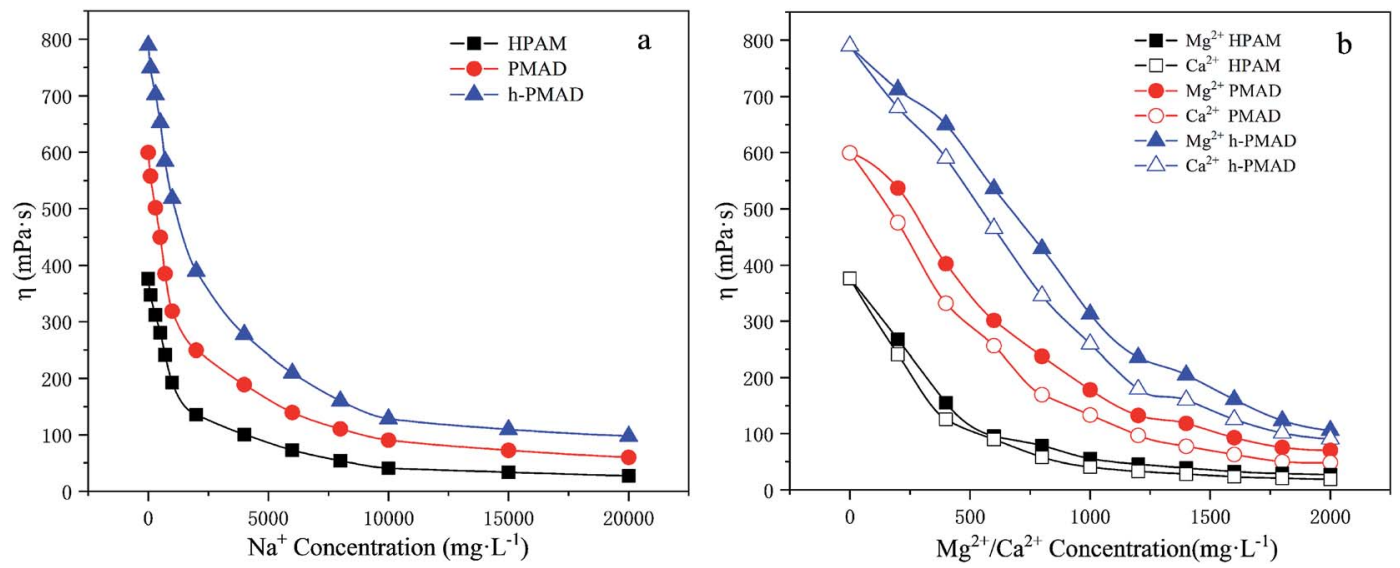

Fig. 8 Salt resistance of polymers for $\mathrm{Na}^{+}, \mathrm{Ca}^{2+}$ and $\mathrm{Mg}^{2+}$.

groups. But in the salt-containing solution, the metal ions $\mathrm{Na}^{+}$, $\mathrm{Ca}^{2+}$ and $\mathrm{Mg}^{2+}$ weaken or shield the mutual attraction between the anion and cationic groups, the molecular chain shows a more stretched state, and so the viscosity of the polymer in the brine drops a little or even increases. As for h-PMAD, the hyperbranched structure improved the spatial density of the zwitterionic monomers, which strengthened the above-mentioned interaction between salts and MMPES, and showed the desired salt resistance.

\subsection{Shear thinning, shear recovery and viscoelasticity}

The effect of the shear rate on the apparent viscosity of the polymer solutions $\left(2000 \mathrm{mg} \mathrm{L}^{-1}\right)$ was researched, as illustrated in Fig. 9(a). It can be observed clearly that the apparent viscosity of the three polymer solutions dropped as the shear rate increased from 7.34 to $80 \mathrm{~s}^{-1}$, and then maintained a small decreasing trend within the shear rate range, showing a nonNewtonian shear thinning behavior. Fig. 9(b), with the shear rate increased from $170 \mathrm{~s}^{-1}$ to $510 \mathrm{~s}^{-1}$ and then returned to 170 $\mathrm{s}^{-1}$ again, shows that the viscosity retention rates of HPAM, PMAD and h-PMAD solutions were $88.66 \%, 94.40 \%$ and $96.33 \%$, respectively. Evidently, the apparent viscosity and viscosity retention of polymer PMAD and h-PMAD solutions are above those of the HPAM all the time, which reveals further the excellent shearing resistance of the two kinds of polymers. More critically, the hyper-branched polymer h-PMAD shows the desired properties in the shear thinning and shear recovery test. For the hyper-branched structure, the molecular chain was entangled, and the strength and toughness of the threedimensional network structure re-associated after dissociation was stronger than that of HPAM. Also, the synergy of hydrogen bonding, hydrophobic association, and electrostatic forces in the polymer solution made the increase in viscosity. ${ }^{54}$ As for a higher viscosity retention, the high shear rate did not destroy the network structure completely, and molecular chains could form small-scale molecular chain micelles with each other. Meanwhile, the high contact probability between the molecular chains helped to form a certain size of fluid mechanics groups; when the shear rate reduced, the dissociated molecular chains were re-associated to form hydrophobic domains and exhibited a recoverable viscosity, in agreement with the literature. ${ }^{55}$

The viscoelasticity of the polymers plays a crucial role in practical application processes, as the high viscoelasticity helps to increase the carrying capacity of the displacement cavities to the residual oil residue and wall oil film. The viscoelasticities of HPAM, PMAD and h-PMAD solutions $\left(2000 \mathrm{mg} \mathrm{L}^{-1}\right)$ were studied via the obtained viscous modulus $\left(G^{\prime \prime}\right)$ and elastic modulus $\left(G^{\prime}\right)$, as shown in Fig. 9(c). It can be seen intuitively that both $G^{\prime}$ and $G^{\prime \prime}$ increased gradually within the oscillation frequency range, in addition, both $G^{\prime}$ and $G^{\prime \prime}$ of PMAD and hPMAD are higher than that of HPAM. Moreover, the curved intersection of h-PMAD has a lower frequency at $0.12 \mathrm{~Hz}$ than those of PMAD at $0.46 \mathrm{~Hz}$ and HPAM at $1 \mathrm{~Hz}$. In the low-
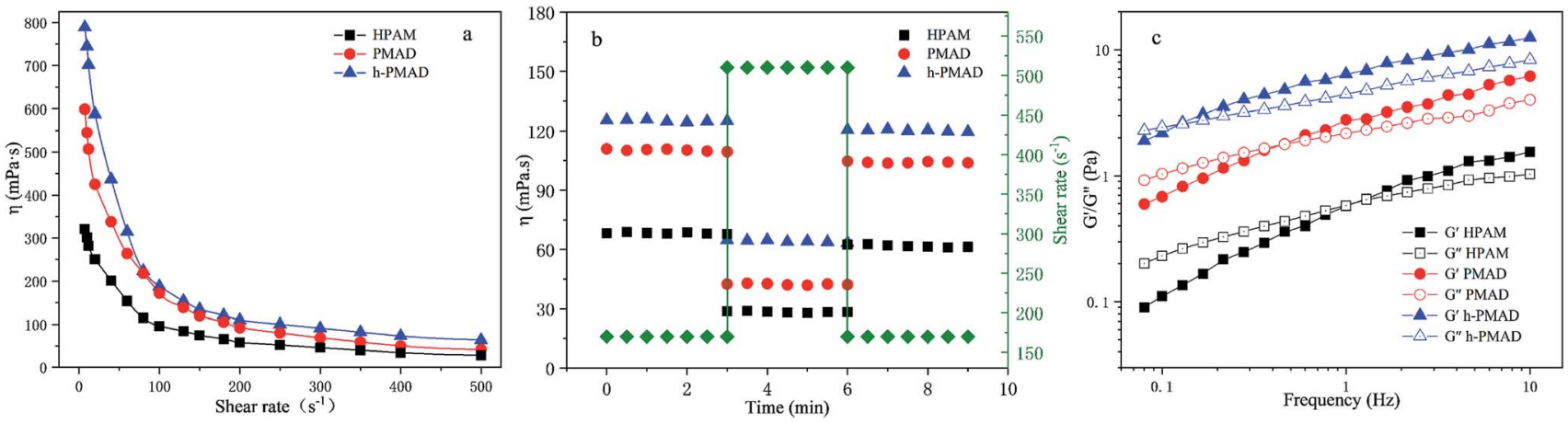

Fig. 9 Rheological behavior of polymers: (a) shear thinning, (b) shear recovery, (c) viscoelastic. 
frequency region where molecules have sufficient time to keep relaxed under dynamic oscillation, ${ }^{56}$ the h-PMAD sample has a good chance to form large-sized aggregates relying on its hyper-branched structure and interactions between the polymer molecules, with the aggregate stored high elastic modulus under stress suggesting the dominance of elastic behavior. ${ }^{57}$

\subsection{Temperature resistance and long-term stability}

The influence of temperature on the polymer solutions $\left(2000 \mathrm{mg} \mathrm{L}^{-1}\right.$ ) in deionized water and formation water (for details see Table S5 $\dagger$ ) were studied from $30{ }^{\circ} \mathrm{C}$ to $100{ }^{\circ} \mathrm{C}$ under a shear rate of $7.34 \mathrm{~s}^{-1}$; the results are displayed in Fig. 10(a) and (b). There are three similar stages in the curves; in the first stage, the solutions apparent viscosity has a slightly decreased tendency with the rise of temperature until about $65{ }^{\circ} \mathrm{C}$ in deionized water and about $55{ }^{\circ} \mathrm{C}$ in formation water. Then, a rapid reduction in the apparent viscosity was observed before the temperature increased to $90{ }^{\circ} \mathrm{C}$. In the final stage, the change of apparent viscosity became steady with temperature from $90{ }^{\circ} \mathrm{C}$; the polymer h-PMAD solution with a viscosity retention of $33.51 \%$ and $50.96 \%$ in formation and deionized water, respectively, showed the optimal temperature resistance. In addition, the h-PMAD possessed a potential long-term stability with a lower viscosity loss after the solution was in simulated formation water at $80{ }^{\circ} \mathrm{C}$ for 4 weeks; the results are shown in Fig. 10(c).

Hydrophobic association is an endothermic driving process; when the temperature rises, the thermal motion of the ionic group increases, and the interaction between the anion and the cation weaken, causing a stretch of the macromolecular chain, and thereby increasing the viscosity of the solution. However, an increase of temperature intensifies the thermal motion of the hydrophobic and water molecules, causing the breaking of the hydration layer surrounding the hydrophobic groups, which weakens the hydrophobic association. Therefore, the relationship between the viscosity of the associative polymer solution and temperature is the result of competition between the two factors. ${ }^{58}$ In our system, when the temperature is relatively low, the effect of the hydrophobic group resulted in a smaller decrease in viscosity. With an increase of temperature, the association of the hydrophobic groups is strengthened, the movement of water molecules is accelerated, and the viscosityincreasing effect and viscosity-reducing effect are simultaneously present, but the viscosity-reducing effect is more significant, so the viscosity retention rate is greatly reduced. Finally, the slightly reduced viscosity retention is mainly a benefit from the monomer DMBSA, as the ring-shaped rigid groups increased the thermal motion resistance, causing an improvement of the polymer molecular backbone thermal stability. ${ }^{59}$ Besides, the hyper-branched structure provides multiple branches and results in a relatively high content of hydrophobic groups, which causes polymer h-PMAD to have the highest final viscosity retention because the hydrophobic association is an endothermic process.

\subsection{Core flooding experiment}

In order to study the ability of mobility control and the capacity to enhance oil recovery of the polymers, core flooding experiments were carried out and the results are shown in Fig. 11 (details in Table S7 $\dagger$ ). For three different systems, the injection pressure continued increasing with the increases of PV during the water and polymer solutions $\left(2000 \mathrm{mg} \mathrm{L}^{-1}\right)$ injection; after the polymer solution flooding, the injection pressure of the sequential water flooding decreased and tended to steady. However, the pressure and oil recovery in the injection stages of the polymer solutions are obvious different. Generally, the RF value is a measure of mobility control capability, so a higher RF value means a greater seepage resistance in the oil reservoir, which is more favorable to improving the swept volume. From Table $\mathrm{S} 7, \uparrow$ the RF values of the polymers HPAM, PMAD and $\mathrm{h}$ PMAD are 7.92, 10.00 and 14.29, respectively. The higher RF value of the h-PMAD indicates its more excellent mobility control ability and higher sweep volume in porous media. In addition, RRF is an index of the polymer solution used to reduce the permeability of porous media; a higher RRF value implies a greater reduction in the permeability of the porous media from the oil reservoir, which reflects in the contribution to enhance oil recovery. Thus, the polymer h-PMAD with an RFF value of 5.07 shows the more visible advantages than PMAD and HPAM with RFFs of 3.64 and 1.66, respectively, as it benefits from the higher retention rate caused by its unique structure and comprehensive properties.
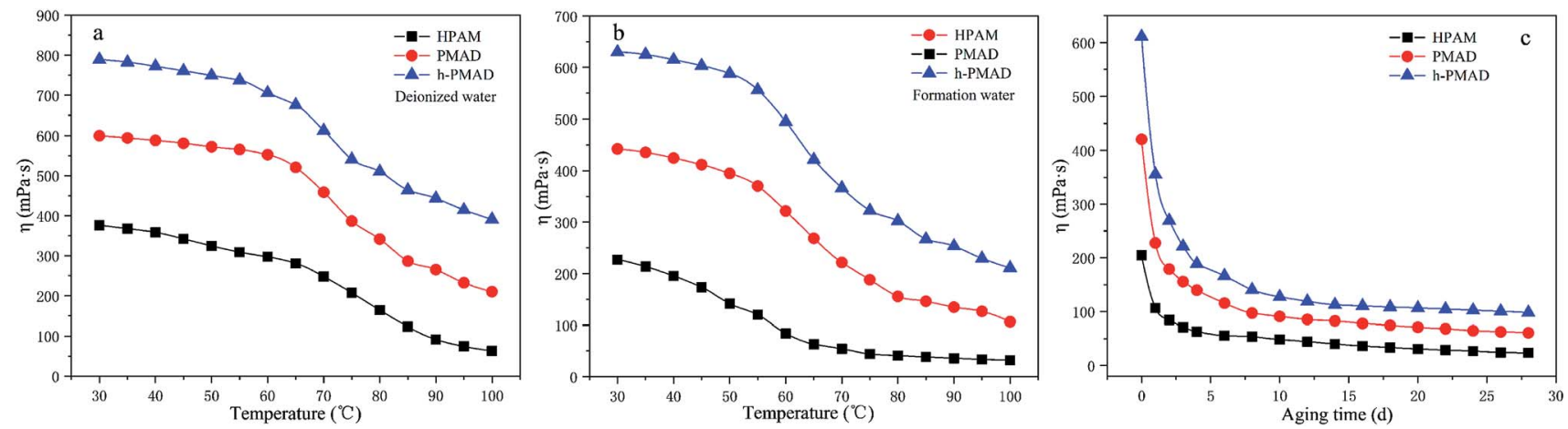

Fig. 10 Rheological behavior of polymers: (a) temperature resistance in deionized water, (b) temperature resistance in formation water, (c) aging in formation water. 

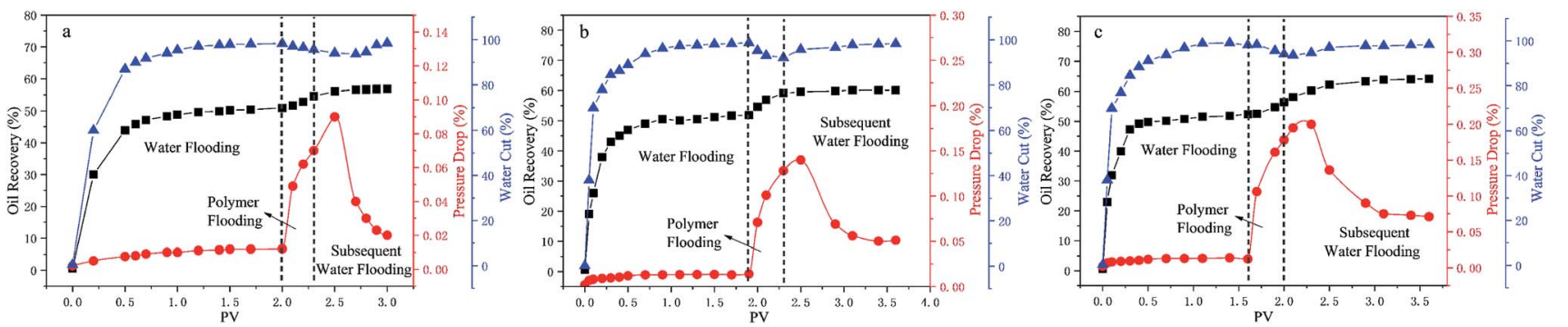

Fig. 11 The core flooding experiments of the polymers: (a) HPAM, (b) PMAD, (c) h-PMAD.

Moreover, according to the experiment results, polymer PMAD with EOR of $8.19 \%$, higher than that of HPAM, 5.88\%, provides powerful evidence that the functionalized polymer has sufficient advantages for this system in polymer flooding. Most importantly, the highest EOR of $11.61 \%$ about h-PMAD reveals that the hyper-branched structure provides an effective carrier for functional monomers to exert a greater effect, which has an extraordinary meaning.

\section{Conclusions}

A hyper-branched structural copolymer h-PMAD with, simultaneously, surface activity, an anti-polyelectrolyte effect and hydrophobic association was successfully prepared via aqueous solution free radical polymerization, and the structure was characterized by IR and NMR. The thermal properties, microscopic appearance, surface activity, thickening ability, rheological properties and aging were compared with those of HPAM and the linear copolymer PMAD, which was prepared using the same major monomers. The results manifested that the hyperbranched copolymer has an excellent comprehensive performance. Also, the copolymer h-PMAD EOR of $11.61 \%$ was obviously higher than PMAD, 8.19\%, and HPAM, 5.88\%. Most importantly, the better EOR of h-PMAD than of PMAD reveals that the hyper-branched structure provides an effective carrier for functional monomers to exert greater effects, which has an extraordinary meaning.

\section{Conflicts of interest}

There are no conflicts to declare.

\section{Acknowledgements}

This work was financially supported by the Key Technology Research and Development Program of Sichuan Province [grant number 2016FZ0121] and the Foundation of Youth Technology Innovation Team of Southwest Petroleum University [grant number 2017CXTD01].

\section{References}

1 D. A. Z. Wever, F. Picchioni and A. A. Broekhuis, Prog. Polym. Sci., 2011, 36, 1558-1628.

2 M. Riahinezhad, L. Romero-Zerón and N. Mcmanus, Fuel, 2017, 203, 269-278.
3 M. J. Caulfield, G. G. Qiao and D. H. Solomon, Chem. Rev., 2010, 33, 274.

4 H. Ehtesabi, M. M. Ahadian and V. Taghikhani, Energy Fuels, 2014, 28, 423-430.

5 M. S. Azad, Y. Dalsania and J. J. Trivedi, Can. J. Chem. Eng., 2018, 96, 2498-2508.

6 S. Arne, Z. Nematollah and G. J. Jacobsen, Colloids Interfaces, 2018, 2, 27.

7 W. L. Kang, L. W. Meng and J. G. Niu, Polym. Mater. Sci. Eng., 2006, 22, 175-177.

8 H. A. Yousefvand and A. Jafari, J. Pet. Sci. Eng., 2018, 162, 283-291.

9 Y. Wang, Z. Y. Lu and Y. G. Han, Adv. Mater. Res., 2011, 4, 306-307.

10 B. I. Choi, M. S. Jeong and K. S. Lee, Polym. Degrad. Stab., 2014, 110, 225-231.

11 M. S. Kamal, A. S. Sultan and U. A. Mubaiyedh, Can. J. Chem. Eng., 2015, 93, 1194-1200.

12 L. Yi, K. Z. Li and D. X. Liu, Adv. Mater. Res., 2013, 800, 411416.

13 A. N. El-Hoshoudy, S. E. M. Desouky and A. M. Alsabagh, Egypt. J. Pet., 2016, 26, S1110062116300332.

14 X. Li, Z. Xu and H. Yin, Energy Fuels, 2017, 31, 2479-2487.

15 T. Li, T. Ci and L. Chen, Polym. Chem., 2013, 5, 979-991.

16 M. Yang, C. Liu and Z. Li, Macromolecules, 2010, 43, 1064510651.

17 R. Liu, W. Pu and J. J. Sheng, J. Taiwan Inst. Chem. Eng., 2017, 80, 639-649.

18 L. Mi and S. Jiang, Angew. Chem., 2014, 53, 1746-1754.

19 Q. Yang and M. Ulbricht, Chem. Mater., 2012, 24, 2943-2951.

20 J. Sun, F. Zeng and H. Jian, Polym. Chem., 2013, 4, 5810-5818.

21 P. Mary, D. D. Bendejacq and M. P. Labeau, J. Phys. Chem. B, 2007, 111, 7767-7777.

22 Z. Chu and Y. Feng, Langmuir, 2012, 28, 1175-1181.

23 C. A. Zuniga, J. B. Goods and J. R. Cox, ACS Appl. Mater. Interfaces, 2016, 8, 1780-1785.

24 M. D. Holtmyer and C. V. Hunt, US Pat. 4959163, 1990.

25 R. Dong, Y. Zhou and X. Zhu, Acc. Chem. Res., 2014, 47, 20062016.

26 D. Wang, T. Zhao and X. Zhu, Chem. Soc. Rev., 2015, 44, 4023-4071.

27 A. K. Patri, I. J. Majoros and J. R. Baker, Curr. Opin. Chem. Biol., 2002, 6, 466-471.

28 S. Hecht, N. Vladimirov and J. M. Frechet, J. Am. Chem. Soc., 2001, 1, 18-25. 
29 Q. Huang, S. Costanzo and C. Das, J. Rheol., 2016, 61, 35-47. 30 D. A. Z. Wever, F. Picchioni and A. A. Broekhuis, Eur. Polym. J., 2013, 49, 3289-3301.

31 W. Pu, R. Liu and B. Li, RSC Adv., 2015, 107, 88002-88013.

32 R. Liu, W. Pu and D. Du, J. Pet. Sci. Eng., 2018, 164, 467-484.

33 B. J. Klemm, F. Picchioni and P. Raffa, Ind. Eng. Chem. Res., 2018, 57, 8835-8844.

34 S. Gou, S. Luo and T. Liu, New J. Chem., 2015, 39, 7805-7814.

35 S. Gou, Y. He and L. Zhou, New J. Chem., 2015, 39, 92659274.

36 S. Gou, Q. Zhang and C. Yang, RSC Adv., 2016, 80, 7669676706.

37 S. Gou, T. Yin and L. Yan, Colloids Surf., A, 2015, 471, 45-53. 38 S. Li, S. Gou and C. Xin, New J. Chem., 2018, 42, 6848-6857. 39 S. Gou, Y. He and Y. Ma, RSC Adv., 2015, 5, 51549-51558.

40 M. Azizi, Eur. J. Org. Chem., 2016, 22, 3796-3803.

41 A. M. S. Maia, R. Borsali and R. C. Balaban, Mater. Sci. Eng. Carbon, 2009, 29, 505-509.

42 D. A. Wever, F. Picchioni and A. A. Broekhuis, Ind. Eng. Chem. Res., 2013, 52, 16352-16363.

43 K. Tsutsumi, Y. Okamoto and Y. Tsukahara, Polymer, 1994, 10, 2205-2209.

44 T. Nakano, A. Matsuda and Y. Okamoto, Polym. J., 1996, 6, 556-558.
45 J. Cao, Y. Tan and Y. Che, J. Macromol. Sci., Part A: Pure Appl. Chem., 2010, 6, 595-601.

46 T. R. Nogueira, M. C. Gonçalves and L. Ferrareso, Adv. Polym. Technol., 2010, 1, 11-19.

47 A. G. And and T. Fukuda, Macromolecules, 1997, 15, 42724277.

48 C. Costa, A. L. Alberton and A. F. Santos, Macromol. React. Eng., 2015, 4, 366-373.

49 L. Yan, T. Yin and W. Yu, RSC Adv., 2015, 5, 42843-42847.

50 M. Alsharif, Doctoral Dissertation, University of Sheffield, 2015.

51 S. Gou, M. Liu and Z. Ye, J. Appl. Polym. Sci., 2014, 8, 631-644. 52 A. Laschewsky and I. Zerbe, Polymer, 1991, 32, 2081-2086.

53 A. N. El-Hoshoudy, S. E. M. Desouky and M. A. Betiha, Fuel, 2016, 170, 161-175.

54 J. Chen, M. Jiang and Y. Zhang, Macromolecules, 1999, 32, 4861-4866.

55 R. A. Muesing, F. A. Lornitzo and S. Kumar, J. Biol. Chem., 1975, 250, 1814-1823.

56 M. Keramatinia, F. Najafi and M. R. Saeb, Prog. Org. Coat., 2017, 113, 151-159.

57 R. Farajzadeh, A. Andrianov and P. L. J. Zitha, Ind. Eng. Chem. Res., 2010, 4, 1910-1919.

58 D. W. Urry, Chem. Phys. Lett., 2004, 399, 177-183.

59 H. Jia and H. Chen, J. Pet. Sci. Eng., 2018, 165, 105-113. 